\title{
A video precipitation sensor for imaging and velocimetry of hydrometeors
}

\author{
X. C. Liu, T. C. Gao, and L. Liu \\ College of Meteorology and Oceanography, PLA University of Science and Technology, Nanjing, China \\ Correspondence to: T. C. Gao (2009gaotc@gmail.com)
}

Received: 15 October 2013 - Published in Atmos. Meas. Tech. Discuss.: 29 November 2013

Revised: 10 May 2014 - Accepted: 12 May 2014 - Published: 9 July 2014

\begin{abstract}
A new method to determine the shape and fall velocity of hydrometeors by using a single CCD camera is proposed in this paper, and a prototype of a video precipitation sensor (VPS) is developed. The instrument consists of an optical unit (collimated light source with multi-mode fibre cluster), an imaging unit (planar array CCD sensor), an acquisition and control unit, and a data processing unit. The cylindrical space between the optical unit and imaging unit is sampling volume $(300 \mathrm{~mm} \times 40 \mathrm{~mm} \times 30 \mathrm{~mm})$. As the precipitation particles fall through the sampling volume, the CCD camera exposes twice in a single frame, which allows the double exposure of particles images to be obtained. The size and shape can be obtained by the images of particles; the fall velocity can be calculated by particle displacement in the double-exposure image and interval time; the drop size distribution and velocity distribution, precipitation intensity, and accumulated precipitation amount can be calculated by time integration. The innovation of VPS is that the shape, size, and velocity of precipitation particles can be measured by only one planar array CCD sensor, which can address the disadvantages of a linear scan CCD disdrometer and an impact disdrometer. Field measurements of rainfall demonstrate the VPS's capability to measure micro-physical properties of single particles and integral parameters of precipitation.
\end{abstract}

\section{Introduction}

The need for detailed knowledge on precipitation microstructure regarding precipitation remote sensing is in the following fields: terrestrial and satellite radio transmission, tropospheric wave propagation, and other atmospheric sciences and applications motivate the continuing development of various disdrometers. Joss-Waldvogel disdrometer (Joss and Waldvogel, 1967; Liu et al., 2013), OTT Particle Size Velocity (PARSIVEL) disdrometer (Löffler-Mang and Joss, 2000), 2-D Video Disdrometer (2DVD) (Kruger and Krajewski, 2002), Hydrometeor Velocity and Shape Detector (HVSD) (Barthazy et al., 2004), and Snowflake Video Imager (SVI) (Newman and Kucera, 2009) are by far the most widely applied instruments for precipitation microstructures, but each instrument has its own advantages and limitations due to its measurement principle.

The Joss-Waldvogel disdrometer infers the size of each raindrop from the measured impact kinetic energy of the raindrops based on an empirical nonlinear assumption between fall velocity and raindrop diameter (Joss and Waldvogel, 1967, 1977), but it cannot measure the velocity and shape of raindrops. OTT PARSIVEL disdrometer can measure the horizontal size of particles by using a decrease of light signal by extinction; the vertical size and fall velocity of particles are estimated by the empirical assumption of raindrops' shape (Löffler-Mang and Joss, 2000), but the difference between assumption and real rainfall can cause a distortion of raindrop size and velocity distribution. And it cannot measure particle shape (Battaglia et al., 2010).

Both 2DVD (Schönhuber et al., 2007) and HVSD (Barthazy et al., 2004) have two line-scan cameras, the vertical velocity of each drop can be calculated according to its travelling time and the distance between two light sheets. 2DVD can measure three-dimensional raindrop shape information, while HVSD can only measure two-dimensional raindrop shape information. Since the raindrop image is acquired in sequential planar sections as it falls through the light sheet, horizontal motion of the droplet in the presence of horizontal winds resulting in a distorted image of 


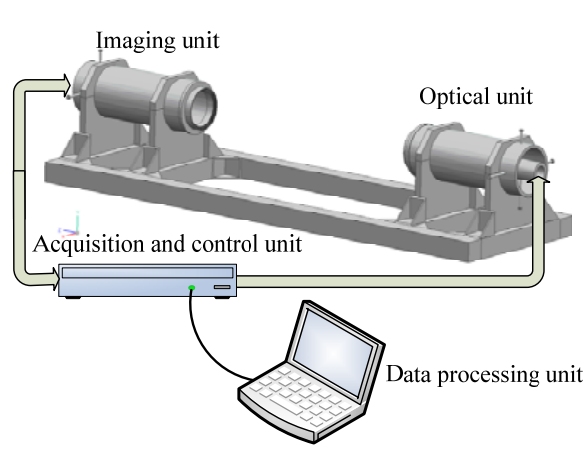

(a)

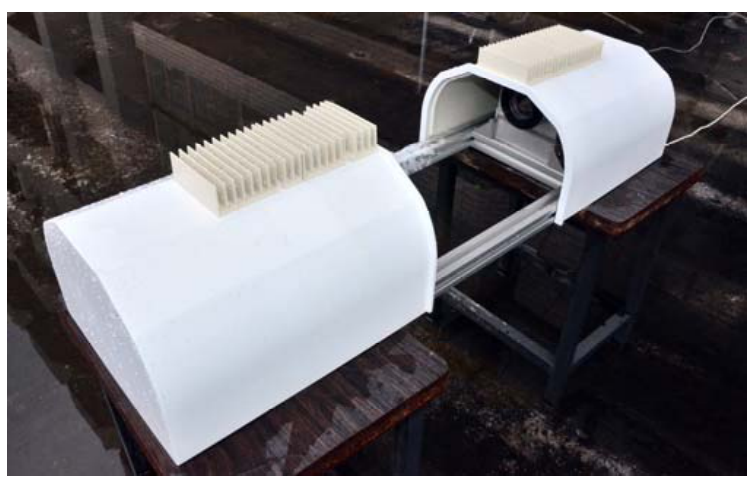

(b)

Figure 1. The Framework of VPS equipment and a photograph of the prototype VPS.

the raindrop. While corrections for this exist, errors in the drop shape measurement remain (Tokay et al., 2001; Thurai and Bringi, 2005; Marzuki et al., 2013). SVI can measure the shapes and size distributions of snowflakes by using a CCD image sensor illuminated by a halogen flood lamp (Newman and Kucera, 2009). Rain Imaging System (RIS) used the similar principle (Saylor et al., 2002; Jones et al., 2003; Saylor and Sivasubramanian, 2007), but they cannot measure the fall velocity of snowflakes/raindrops.

This paper presents a new ground-based video precipitation sensor (VPS) for imaging and velocimetry of hydrometeors; it can measure the shape, size, orientation, and fall velocity of naturally falling hydrometeors simultaneously by using a planar array CCD camera with its exposure controlled precisely, which represents a promising alternative to monitor precipitation particles. From these, rain rate, rain accumulation, drop size distribution, drop velocity distribution, and other precipitation-related variables can be estimated. The following sections contain descriptions of the instrument VPS, of the image processing and feature extraction algorithms, of the results of calibration and field experiments. The last part summarizes the main features and future work of VPS.

\section{The VPS system}

\subsection{Main components}

The VPS consists of four units: optical unit, imaging unit, acquisition and control unit, and data processing unit, as shown in Fig. 1a. The optical unit and imaging unit are integrated into one tunnel housing, on which there are a couple of metal splash elimination grids, to prevent raindrops splashing into the sampling area and to minimize the wind disturbances about the instruments, as shown in Fig. $1 b$.

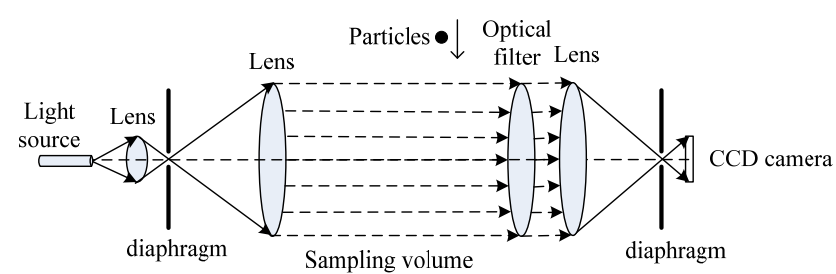

Figure 2. The schematic diagram of imaging.

The optical unit contains a multi-mode fibre cluster, expanded beam lens, and concentration lens, which provide a parallel light beam for imaging unit; considering that small particles illuminated by laser light will create diffraction patterns, the incoherent light $(\lambda=460 \mathrm{~nm})$ from LED is used in this system, and the multi-mode fibre cluster are used to concentrate the light and increase the light intensity. To address the depth of field in the imaging system, we design a telecentric afocal imaging system, as shown in Fig. 2. The chief ray is parallel to the axis in object space and image space, and both the EP (Entrance Pupil) and the XP (Exit Pupil) are located infinitely far from the lens, which ensure that the image size does not change with focus position. The centred lenses are used to generate the parallel light beam, the divergence is less than $0.2 \mathrm{mrad}$.

The imaging unit contains one charge-coupled device (CCD) image sensor and matching driving circuit, the CCD image sensor has 640 pixels $\times 480$ pixels in interlaced mode; we operated it in 640 pixels $\times 240$ pixels non-interlaced mode (50 frames per second). The single exposure time is $20 \mu$ s so blurring that particle motion is insignificant, and the time between two exposures is $2 \mathrm{~ms}$ so that the two exposures of the same particle can be recorded in one image. The superiority of a CCD camera is that images can be obtained almost instantaneously and thus there's no need to correct the particle movement. 


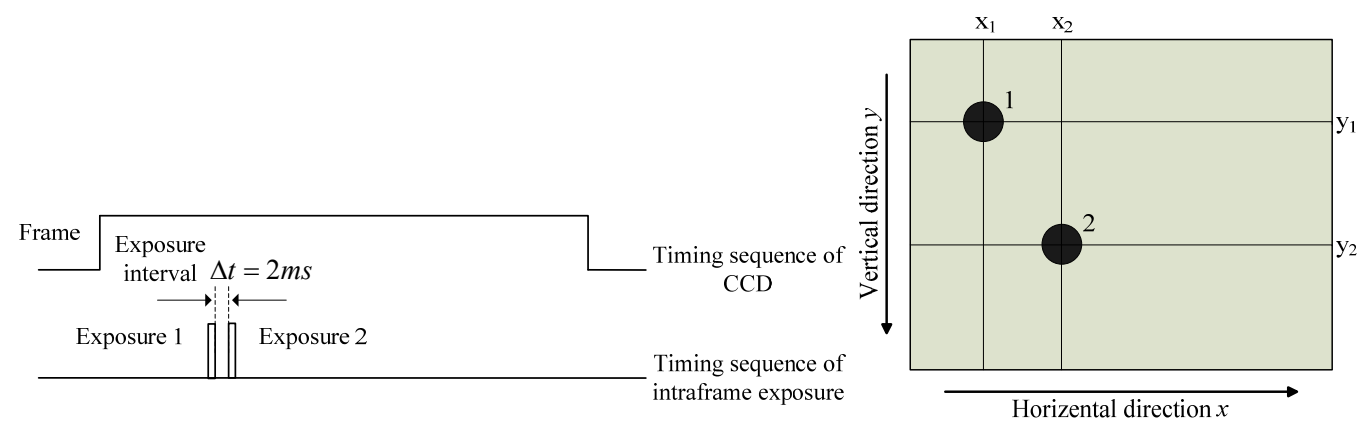

Figure 3. The timing sequence of exposure and images recorded by VPS.

The acquisition and control unit contains a digital signal processor (DSP, C6000 series, TMS320DM642), a complex programmable logic device (CPLD, MachX0 series, LCMX0256), Ethernet driver chips (LXT971ALC), reset circuit, clock circuit, storage chips and other support integrated circuits. The acquisition and control unit controls the optical unit and imaging unit, records and preprocesses the raw images, encodes and transmits the raw data. The data processing unit is a PC terminal that communicates with the acquisition and control unit using a coaxial network cable via the internet TCP/IP protocol. Software running on the terminal receives the data obtained by the sensor, processes the hydrometeors' images, and calculates the size, velocity, and shape of hydrometeors.

\subsection{Principle of operation}

The optical unit generates a cylindrical light beam that is projected onto the CCD image sensor with a special driving circuit. The light beam is so bright that the VPS is insensitive to ambient light. Precipitation particles falling through the light beam shadow are rendered opaque and are then recorded by the CCD camera. The images are then compared against a gray scale threshold (determined by calibration) to determine whether each pixel of images is lit or obscured. The CCD camera has a resolution of $640 \times 240$, the corresponding field of view is $40 \mathrm{~mm} \times 30 \mathrm{~mm}$, and hence each pixel size of an image is $0.0625 \mathrm{~mm}$ (horizontal) $\times 0.125 \mathrm{~mm}$ (vertical). The CCD camera runs in 50 frames per second, a pulse synchronous signal generator is used to generate two exposures in each frame, the interval of two exposures is $2 \mathrm{~ms}$. the timing sequence of exposure and images recorded by VPS are shown in Fig. 3.

The CCD camera records the double-exposure image of each particle in a single frame, by which the two-dimensional particle shape information can be obtained, and the size, volume, equivalent diameter, as well as oblateness of particles can be calculated by the image processing algorithm. Each particle is exposed twice in a single frame, its displacement can be calculated according to the location of two exposed images, hence the vertical velocity and horizontal velocity component in the plane (say $X-Z$ ) of each particle can be calculated according to its displacement and exposure interval. It should be noted that the component of velocity, axis ratio, and canting angle in the $Y-Z$ plane cannot be measured due to the two-dimensional imaging system by one camera, and the horizontal velocity of drops determined by only one camera is only one vector of true horizontal velocity in one projection. However, the raindrops are essentially rotationally symmetric (Huang et al., 2008), therefore the 2-D imaging of long-time sampling can describe the 3-D drops. The drop size distribution and drop velocity distribution can be obtained by the time integration, by which the intensity, amount, and type of precipitation can be estimated.

Preliminary experiments found that there are certain noises in the background of the image due to the optical lens polluted by dust, dew, or frost in the natural environment, light intensity might change due to the instability of the light source in the long-time running, therefore a real-time adaptive averaging method is designed to remove the background of the image, which has been embedded in the DSP software. Considering that the sampling rate of VPS is 50fps, the maximum data of original images might reach $25.7 \mathrm{~GB}$ per hour, which is a great challenge to the computer storage. To minimize the data sizes, an encoding method for original images is proposed: there are only minimum enclosing rectangles (MER) around the raindrop images, which are saved and transmitted to the data processing unit. The flowchart of background removal, image detection, and encoding is shown in Fig. 4, the details of the process are the following: (i) original images are stored in the cache; (ii) each of the 1024 frame images are averaged as a background of image; (iii) original images are subtracted from the background; (iv) two images of the same individual particle are paired and transmitted to the data processing terminal. If two images have the same gray scale, area, horizontal size, and vertical size, it can be identified as the same particle; otherwise it can be identified that they are from the different particles, and the embedded software does not transmit the incomplete image pair to the data processing terminal. 


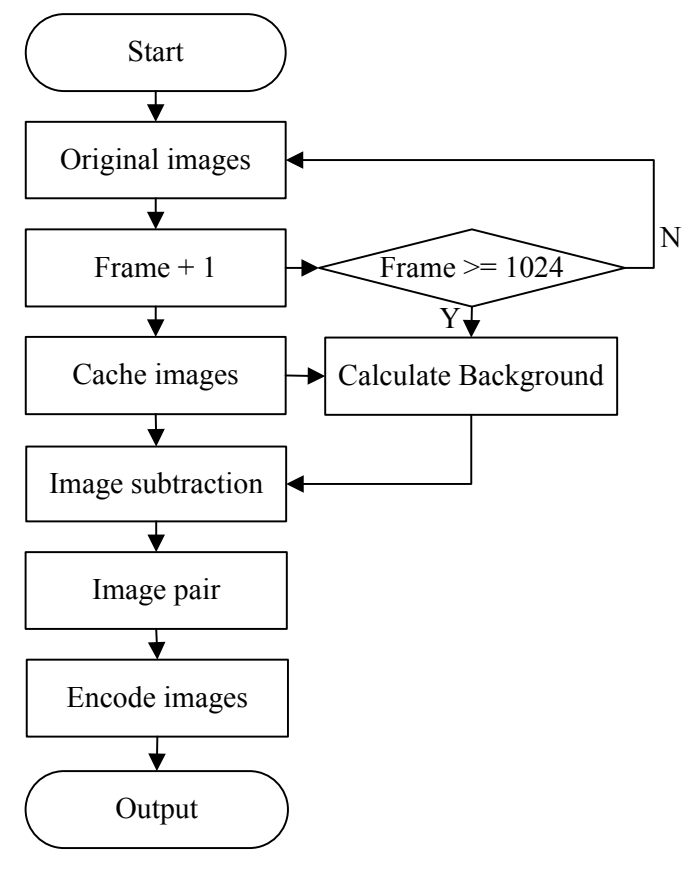

Figure 4. Flowchart of background removal, image detection, and encoding.

\section{Data processing algorithm}

\subsection{Image processing}

The CCD camera has a resolution of $640 \times 240$, the corresponding field of view is $40 \mathrm{~mm} \times 30 \mathrm{~mm}$, and hence the minimum particle $(0.1 \mathrm{~mm})$ has 1.6 pixels in horizontal direction and 0.8 pixels in vertical direction theoretically. Preliminary imaging experiments found that there are image diffusions due to the defocusing of imaging system, which cause the overestimation of particle size. We propose an image restoration algorithm based on point spread function (PSF), considering that the motion blur of particles is insignificant, the defocusing blur is the main factor for images degradation, in which the PSF can be defined as:

$h(i, j)=\left\{\begin{array}{ll}1 /\left(\pi R^{2}\right) & \sqrt{i^{2}+j^{2}} \leq R \\ 0 & \sqrt{i^{2}+j^{2}}>R\end{array}\right.$,

where the $R$ is the defocusing radius.

Figure 5 shows the flowchart of image processing and feature extraction. The image processing begins with a median filter algorithm (step 1), to remove the noise and obtain the filtered images; next the PSF (step 2) is applied to restore the filtered images; then the binarization algorithm (step 3) is used to obtain the binary images; the edge detection algorithm (step 4) is used to detect the edges and obtain the contour of particles; at last the size detection algorithm (step 5) is used to calculate the long axis, short axis, equivolumetric diameter, axis ratio, orientation, and etc. As shown in Fig. 6,

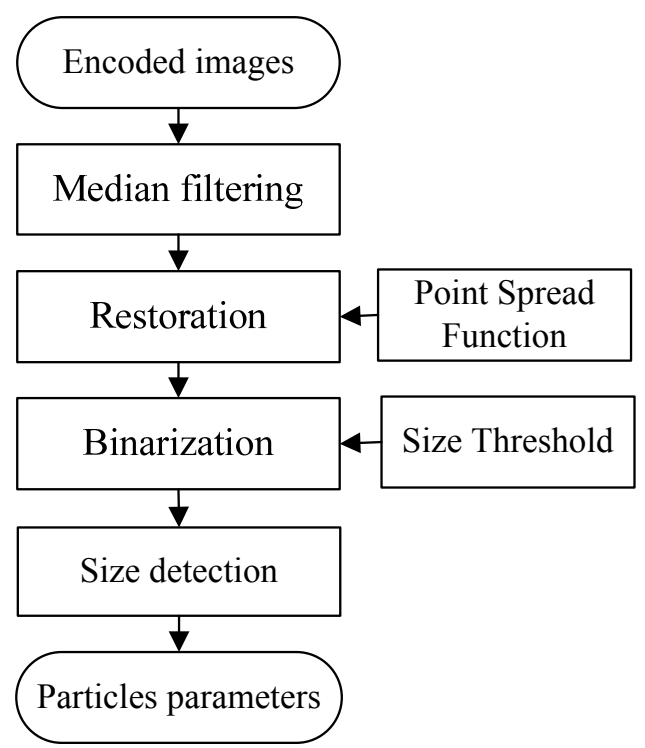

Figure 5. Flowchart of image processing and feature extraction.

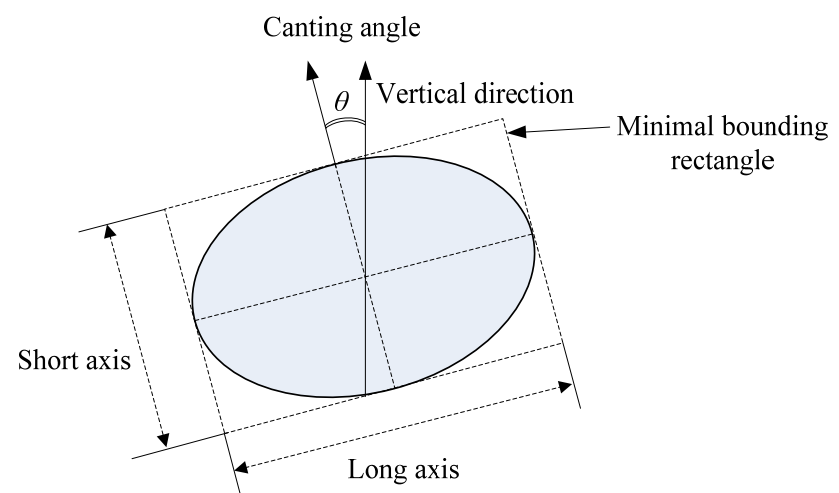

Figure 6. Minimal bounding rectangle of raindrop image and orientation angle.

The long axis and short axis of each drop can be obtained according to the minimum bounding rectangle of its image, by which the axis ratio can be calculated, and the orientation can be calculated. According to the operation principle of 2DVD, the ratio between the maximum horizontal chord and maximum vertical chords has to be corrected for the orientation angles (Thurai and Bringi, 2005; Thurai et al., 2007, 2009a, 2011), whereas the VPS can record the complete images of raindrops by planar CCD camera, the displacement of raindrops during the exposure time can be ignored, therefore the axis ratio can be obtained without correction.

We validated the image processing algorithm using spherical glass balls, which have the similar refractive index with the water drops. Figure 7 shows the results of image processing $(D=2 \mathrm{~mm})$, it can be found that there are fuzzy edges and noise in the original images (Fig. 7a); and the noise can 


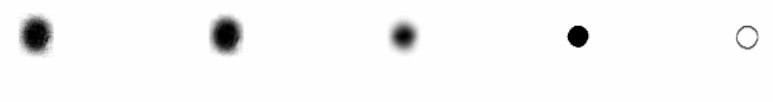

(a) Original images (b) Filtered images (c) Restored images (d) Binary images (e) Particles contour

Figure 7. Images of a glass ball for calibration $(D=2 \mathrm{~mm})$.

be removed by the median filter, as shown in Fig. 7b; the size of restored images in Fig. 7c can be reduced by the restoration of point spread function; the binary image and clear edge of particles can be obtained by the binarization algorithm, as shown in Fig. 7d and e. It can be concluded that this algorithm can obtain the sharp image of particles, based on which the VPS and analysis software can provide precise measurements for the size, shape, orientation, velocity of particles.

\subsection{Feature extraction}

Considering the operation principle of VPS, the effective sampling time is only $10 \%$ of the exposure time of each frame; therefore not every particle that passes through the sampling area can be double-imaged by camera. The probability that a certain particle with a certain size and fall velocity can be fully photographed twice by the camera in the vertical dimension and the horizontal dimension is defined as (so-called capture probability)

$$
\left\{\begin{array}{l}
\text { Prob }_{\text {vertical }}=\frac{H-D_{\mathrm{V}}-V\left(D_{\text {eq }}\right) \cdot T_{\text {interval }}}{V\left(D_{\text {eq }} \cdot 20 \mathrm{~ms}\right.} \\
\text { Prob } \\
V_{\text {horizontal }}=\frac{W-D_{\mathrm{H}}-V_{\mathrm{H}} \cdot T_{\text {interval }}}{V_{\mathrm{H}} \cdot 20 \mathrm{~ms}}
\end{array},\right.
$$

where $H$ is the height of sampling size, $D_{\mathrm{V}}$ is the vertical size of drops, $V\left(D_{\text {eq }}\right)$ is the average velocity for each particle size bin with equivalent diameter, $T_{\text {interval }}$ is the exposure interval in each frame, and $20 \mathrm{~ms}$ is the exposure time of each frame. $W$ is the width of sampling size, $D_{\mathrm{H}}$ is the horizontal size of drops, and $V_{\mathrm{H}}$ is the horizontal velocity of drops.

Figure 8a shows the capture probability of different raindrops with different fall velocities in the vertical dimension. It can be found that the capture probability of a raindrop with the diameter $1 \mathrm{~mm}$ and fall velocity $4.3 \mathrm{~m} \mathrm{~s}^{-1}$ is $20 \%$, the capture probability of a raindrop with the diameter $3 \mathrm{~mm}$ and fall velocity $8 \mathrm{~m} \mathrm{~s}^{-1}$ is $5 \%$, and when the diameter of raindrop is larger than $7.8 \mathrm{~mm}$, the capture probability is below $0 \%$, which means that raindrops larger than $7.8 \mathrm{~mm}$ cannot be fully photographed twice. It can be refined by shortening the exposure interval or enlarging the sampling area. Figure $8 \mathrm{~b}$ shows the capture probability of different raindrops with different horizontal velocity in the horizontal dimension, it can be found that the horizontal capture probability of raindrop decreases with the increasing of the diameter and horizontal velocity. The horizontal capture probability of a raindrop with the diameter $10 \mathrm{~mm}$ and horizontal velocity $10 \mathrm{~m} \mathrm{~s}^{-1}$ is $3.5 \%$. It should be noted that the limitation of

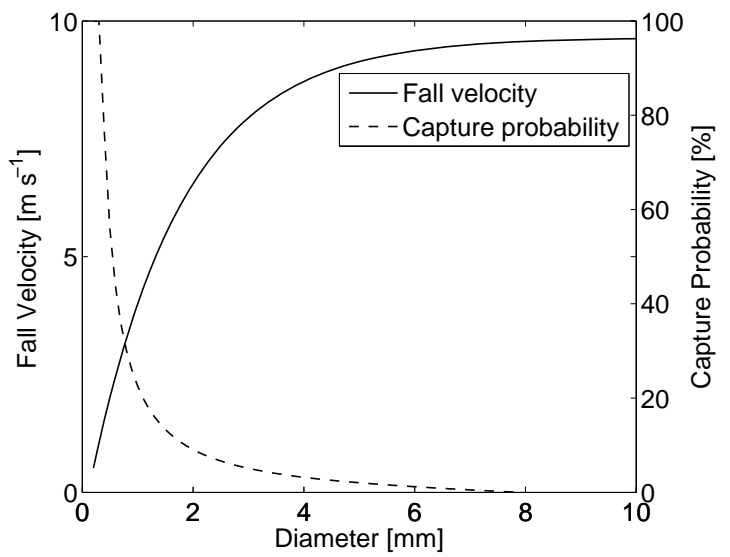

(a) Capture pobability in vertical dimension

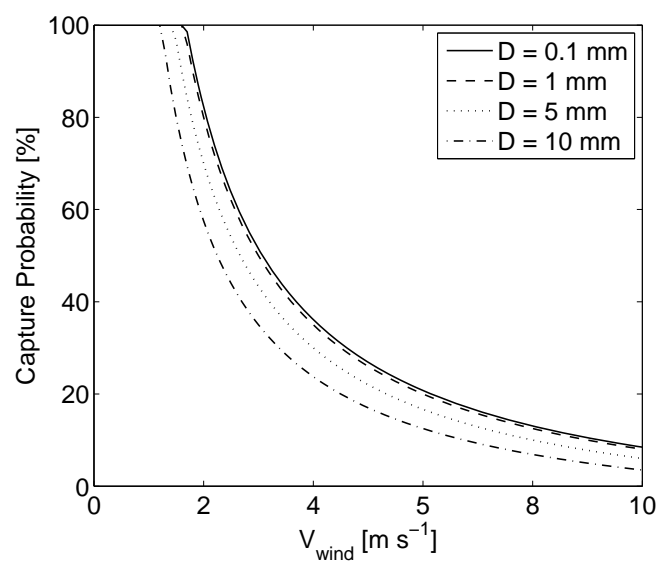

(b) Capture probability in horizontal dimension

Figure 8. Capture probability of different raindrops in the vertical dimension and horizontal dimension $\left(T_{\text {interval }}=2 \mathrm{~ms}\right)$.

capture probability can be complemented by long-term sampling.

The raindrop size distribution $N\left(D_{\text {eq }}\right)$ can be computed as follows:

$N\left(D_{\text {eq }}\right)=\frac{\operatorname{Num}\left(D_{\text {eq }}\right)}{A_{\text {sampling }} \cdot \mathrm{d} D_{\text {eq }} \cdot \operatorname{Prob} b_{\text {vertical }} \cdot \text { Prob horizontal }}$,

where $N\left(D_{\mathrm{eq}}\right)$ is the number concentration of particles per volume per size $\left(\mathrm{m}^{-3} \mathrm{~mm}^{-1}\right) ; \operatorname{Num}\left(D_{\text {eq }}\right)$ is the sampled number of particles of each bin, which can be obtained by counting particles and summing volume increments during the time interval ( $1 \mathrm{~min}$ in this system); $A_{\text {sampling }}=300 \mathrm{~mm} \times\left(40 \mathrm{~mm}-D_{\mathrm{H}} / 2\right) \times$ (30 $\mathrm{mm}-D_{\mathrm{V}} / 2$ ), which is the equivalent sampling volume; $\mathrm{d} D_{\text {eq }}$ is the interval size of particle, which is equal to $0.2 \mathrm{~mm}$ for 50 bins spanning from $0.1 \mathrm{~mm}$ to $10 \mathrm{~mm}$; Prob ${ }_{\text {vertical }}$ and 


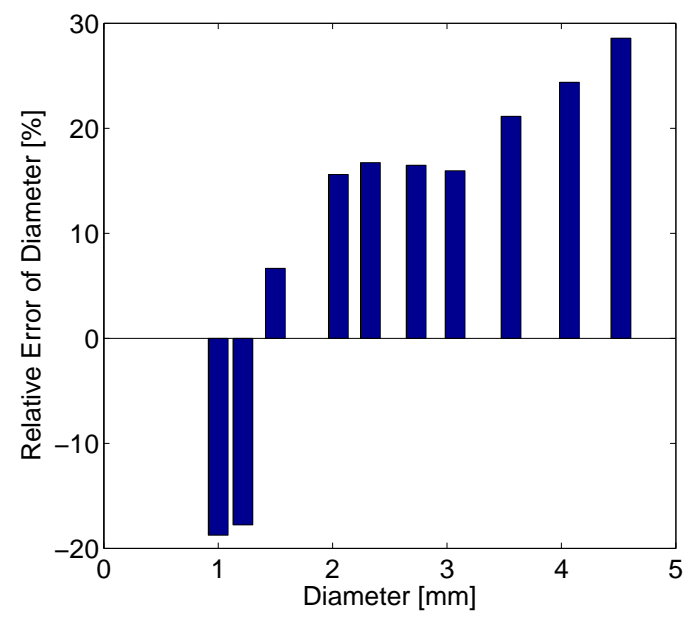

Figure 9. Relative error of diameter versus diameter (threshold $=3 \%$ ).

Prob $_{\text {horizontal }}$ are the vertical and horizontal capture probabilities of a raindrop with equivalent diameter $D_{\text {eq }}$.

Rain rate $(R)$ can be calculated by the integration of drop size distribution $N\left(D_{\text {eq }}\right)$, velocity distribution $V\left(D_{\text {eq }}\right)$, equivolumetric diameter $D_{\text {eq }}$, and density $\rho$ :

$$
R=\frac{\pi}{6} \int_{0}^{\infty} N\left(D_{\mathrm{eq}}\right) \rho D_{\mathrm{eq}}^{3} V\left(D_{\mathrm{eq}}\right) \mathrm{d} D_{\mathrm{eq}}
$$

\section{Calibration}

The VPS fundamentally takes photographs of precipitation particles when they pass through the sampling volume; it infers horizontal and vertical dimensions of particles from the images, and the estimation of particle velocity is independent from the particle shape, therefore the size of particles are the only required data for calibration. The telecentric imaging lens designed in VPS infers that the depth of field (DOF) of VPS is infinite; therefore a single particle falling at different distances from the centre of focus can be recorded as the same size of images. However, the light source in VPS system is not the ideal point light source, the defocusing blurred images are larger than the real sizes; therefore a proper defocusing radius of PSF and a binarization threshold are crucial to the successful operation of VPS.

Preliminary test of the point light source has determined that the defocusing radius of PSF is 5 pixels; hence the determination of gray scale threshold is a critical part of calibration. It is clear that if the binarization threshold is high, the VPS underestimates particle sizes; whereas if the threshold is low, the VPS overestimates the sizes. In this paper, the VPS is calibrated by dropping small glass balls (12 sizes ranging from $1 \mathrm{~mm}$ to $5.8 \mathrm{~mm}$ in diameter) through the sampling area.

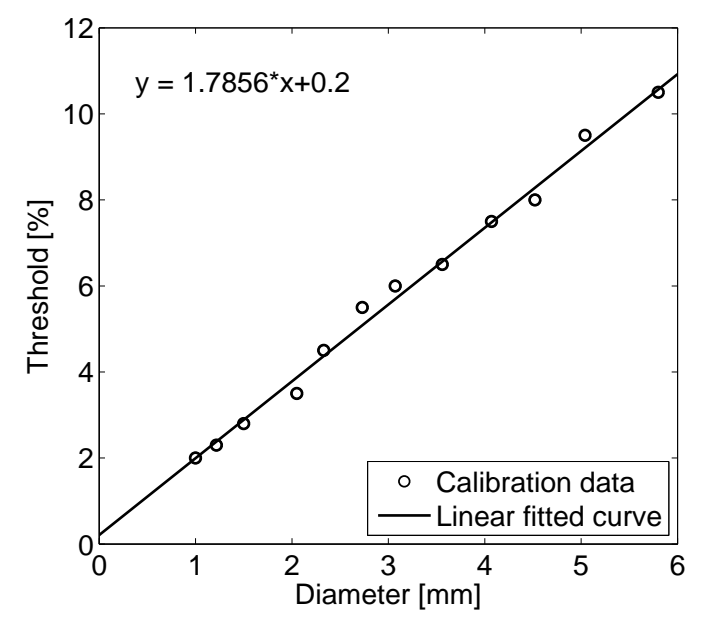

Figure 10. Calibrated binarization threshold for different diameters.

Figure 9 shows the result of calibration images by a fixed threshold ( $3 \%)$; note that if we set a fixed gray scale threshold to the different size of particles, the sizing error of particles would increase and the error of rain rate and accumulated rain amount would increase. Limited by the samples of ideal spherical glass ball, the calibration glass ball does not cover the size range of rain drops, therefore we create a look up table of threshold by using the linear least square fitting method, which ranges from $0 \mathrm{~mm}$ to $6 \mathrm{~mm}$, the results are shown in Fig. 10. The particles with different size can be binarized with different threshold, so that the sizing error of VPS is suitable for precipitation measurement and routine operation.

\section{Field measurement}

A VPS system was located at Nanjing, China, during May 2013, by which several rainfall events have been observed. 39218 frames of images were recorded from 23:00 to 24:00 on 8 May 2013 (UTC), in which 6317 images of raindrops were recorded, and hence the overall capture probability is $16.1 \%$.

Figure 11 shows the distributions for fall velocity (Fig. 11a), axis ratio (Fig. 11b), and canting angle (Fig. 11c) of raindrops. Considering that the rain rate is only $2.5 \mathrm{~mm} \mathrm{~h}^{-1}$ (measured by rain gauge), this rainfall is mainly dominated by small drops $\left(D_{\text {eq }}<2 \mathrm{~mm}\right.$ ), and the maximum diameter is $2.3 \mathrm{~mm}$. The fall velocity of raindrops increases obviously with the increasing of the diameter, and the observed data are in good agreement with the empirical velocity relationship proposed by Atlas (Atlas et al., 1973). It should be noted that there are a certain number of velocity outliers of raindrops measured by the VPS, in which some large raindrops have small velocities, and some small raindrops have large velocities beyond empirical values. The 


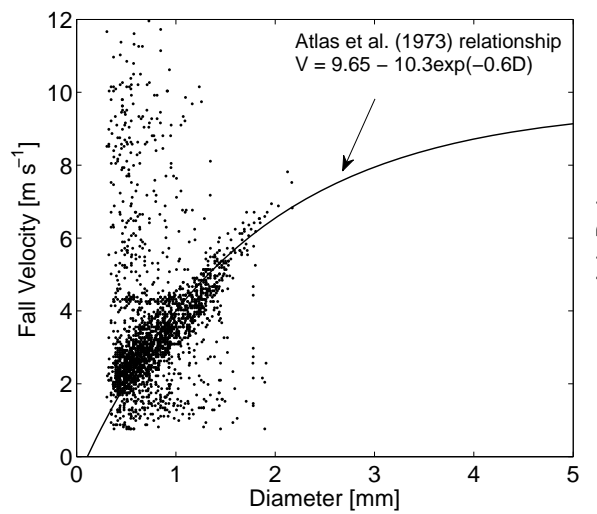

(a)

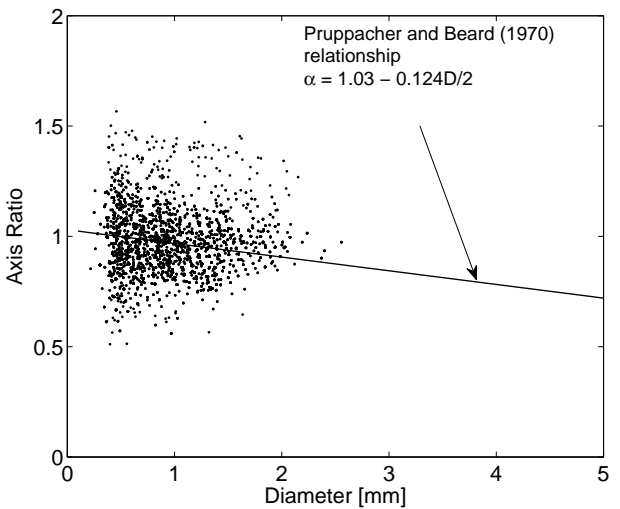

(b)

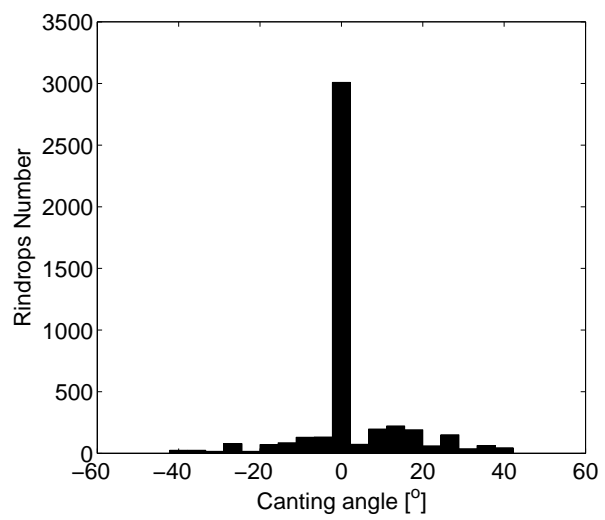

(c)

Figure 11. Fall velocity, axis ratio, and canting angle of raindrops observed by VPS.

possible reason is that there was a strong wind with speed $>8 \mathrm{~m} \mathrm{~s}^{-1}$, wind blow, turbulence, and splashing where one drop hits the side of VPS and a part of it passes through the measuring area might causes the deviation of normal fall velocity. And another possible explanation is that the different particles with the same size might be identified as the same particle by the image pair algorithm, the outliers of fall velocity might occur on this occasion.

Many researchers have studied the relationships between the axis ratio and the diameter of raindrops by theoretical analysis, laboratory measurement and field measurement; several relationships have been proposed (Beard and Chuang, 1987; Chandrasekar et al., 1988; Andsager et al., 1999; Thurai et al., 2009b; Marzuki et al., 2013; Thurai et al., 2007). The observed axis ratio of large raindrops has an obvious correlation with the empirical relationship by Pruppacher and Beard (Pruppacher and Beard, 1970), while the axis ratio of small raindrops shows a much wider dispersion. Considering that the axis ratio of small raindrops $\left(D_{\text {eq }}<1 \mathrm{~mm}\right)$ is greater than 0.96 , the axis ratio of large raindrops is the focus of research. We will observe more large raindrops and study the axis ratio distribution of raindrops in a future field experiment.

The canting angle (so-called orientation) is the angle between the projection of the drop's rotational symmetry axis and the projection of the "true" local vertical direction, which has a certain influence on the polar radar measurements (Huang et al., 2008). Figure 11c shows the canting angle distribution in one projection. Note that the mean canting angle is nearly $2.1^{\circ}$, with the standard deviation close to $11.5^{\circ}$, which is greater than the canting angle from the side-view of 80-m fall artificial rainfall with the 2DVD; the possible reason is that there was a strong wind (stronger than $8 \mathrm{~m} \mathrm{~s}^{-1}$ ) at that time, causing a much wider variation.

The VPS recorded a rainfall event from 21:00, 8 May 2013 to $04: 00,9$ May 2013 (UTC); the time history of raindrops number per minute, rain rate, raindrop size distribution, and rain accumulation are shown in Fig. 12. During the event, the VPS recorded 157694 frames of images, in which there were 26566 images of raindrops; the average 


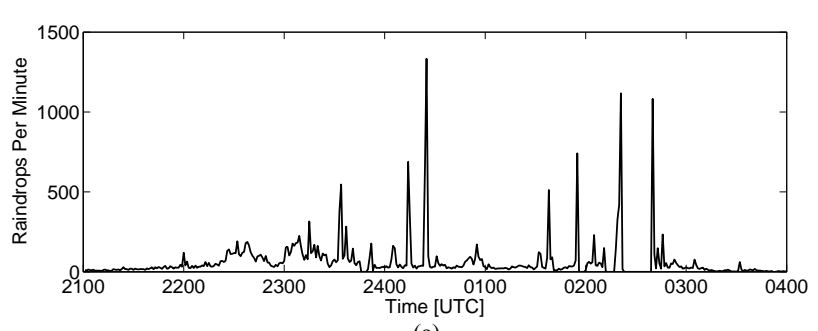

(a)

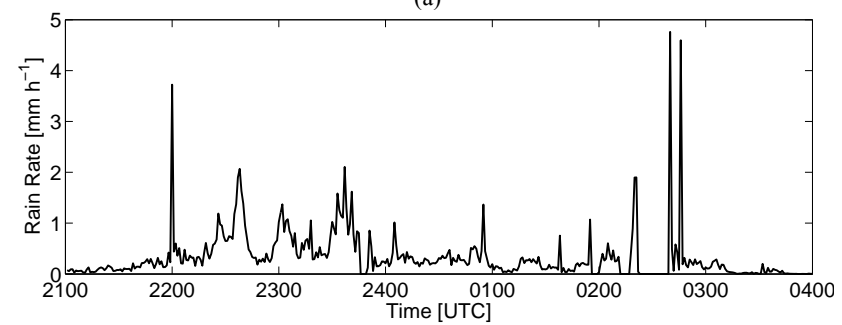

(b)

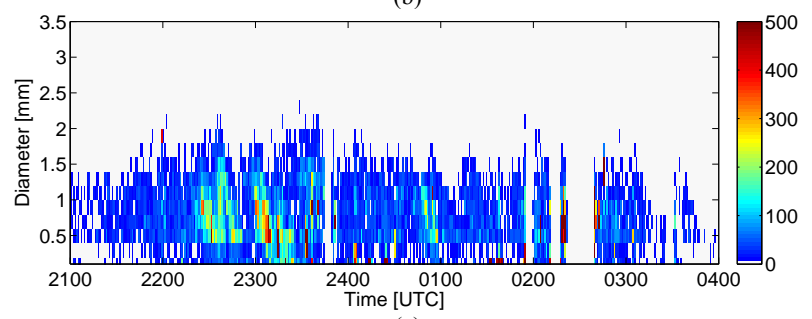

(c)

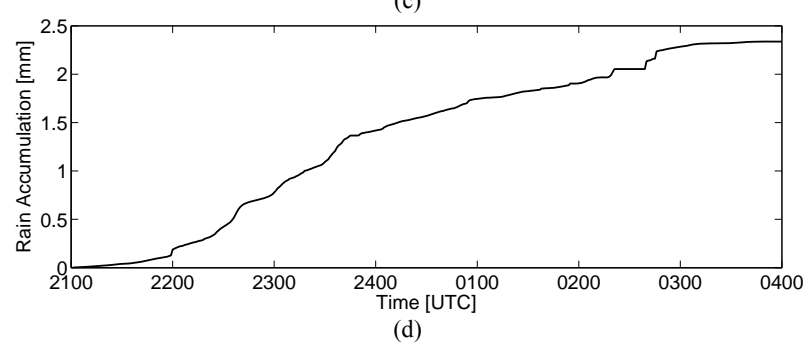

Figure 12. History of rainfall observations from 21:00, 8 May 2013 to 04:00, 9 May 2013 (UTC).

is about 63 raindrops per minute, and overall capture probability is $16.8 \%$. The VPS frame rate is $50 \mathrm{fps}$, so about 1 in 47 frames had a couple of double-exposure raindrops. We conclude that the VPS yields a sufficient number of particles for hydrometeors micro-physical characteristics studies. As shown in Fig. 12a and b, the maximum raindrops number per minute was 1333 occurred during 00:25 UTC, whereas the rain have not the strongest rain rate, the reason is that small raindrops are in the majority, which contribute little to rain rate, it can be validated by the Drop Size Distribution in Fig. 12c. Figure $12 \mathrm{~d}$ shows that the the total rain accumulation is $2.34 \mathrm{~mm}$, whereas the rain gauge reported a total rain amount of $2.5 \mathrm{~mm}$, hence the overall error is $0.16 \mathrm{~mm}$.

An OTT PARSIVEL disdrometer was installed from June 2013, based on which the rain rate, rain accumulation, drop size distribution, and fall velocity are compared. Figure 13 shows the results of a rainfall event from 14:00 to 17:00, 7 June 2013 (UTC). During the event, the VPS recorded 69896 frames of images, in which there were 7821 images of raindrops, hence the overall capture probability is $11.2 \%$. Figure 13 a shows that the correlation coefficient of rain rate by VPS and OTT is $80.3 \%$, and the standard deviation is $1.1 \mathrm{~mm} \mathrm{~h}^{-1}$, the maximum rain rate recorded by VPS and OTT is $6.4 \mathrm{~mm} \mathrm{~h}^{-1}$ and $3.3 \mathrm{~mm} \mathrm{~h}^{-1}$. Figure $13 \mathrm{~b}$ shows that the correlation coefficient of rain accumulation by VPS and OTT is $99.1 \%$, and the standard deviation is $0.13 \mathrm{~mm}$, the rain accumulation recorded by VPS and OTT is $0.73 \mathrm{~mm}$ and $0.74 \mathrm{~mm}$.

Note that the number of outliers of fall velocity might be quite large. If the velocity of a raindrop is outside the following range, it would be identified as an outlier and removed:

$\left|v_{\text {measured }}-v_{\mathrm{a}}\right|<0.4 v_{\mathrm{a}}$,

where $v_{\mathrm{a}}$ is the Atlas (Atlas et al., 1973) fall velocity relationship.

Figure $13 \mathrm{c}$ and $\mathrm{d}$ present the fall velocity of raindrops measured by the VPS and OTT disdrometer. The velocity resolution of VPS is $0.2 \mathrm{~m} \mathrm{~s}^{-1}$, whereas the velocity resolution of OTT decreases with the increases of size. It can be found that the fall velocity of raindrops increases exponentially with the increase of size. The comparison of VPS and OTT shows that both the number of raindrops and velocity distribution are in good consistency, except for that there are a few smaller raindrops with higher velocity recorded by OTT. The possible reason is that there might be some small raindrops splashed by large raindrops with higher velocity passing through the sampling area, and no velocity correction applies to OTT data. Figure 13e shows that the drop size distribution obtained by VPS is quite consistent with that obtained by OTT, except for that there are a few discrepancies for the small drops $(D<0.3 \mathrm{~mm})$. It should be noted that only light rainfall events are considered in this case, the accuracy of VPS in heavy rainfall remains to be examined in the future.

\section{Conclusions}

An innovative ground-based optical instrument, the video precipitation sensor (VPS), was developed to measure the detailed information of free falling precipitation particles in a natural environment, which provides a promising alternative to monitoring precipitation particles. It is a low-cost instrument and requires minimal maintenance for long-lasting running in the field.

The VPS has one horizontal cylindrical parallel light beam $(300 \mathrm{~mm} \times 40 \mathrm{~mm} \times 30 \mathrm{~mm})$, the corresponding sampling volume is $360 \mathrm{~cm}^{3}$ and the horizontal sampling area is $120 \mathrm{~cm}^{2}$, the double-exposure images of individual precipitation particles in free fall can be recorded by a planar array CCD camera, the size, shape, orientation are determined from the images, the fall velocities are determined from the 


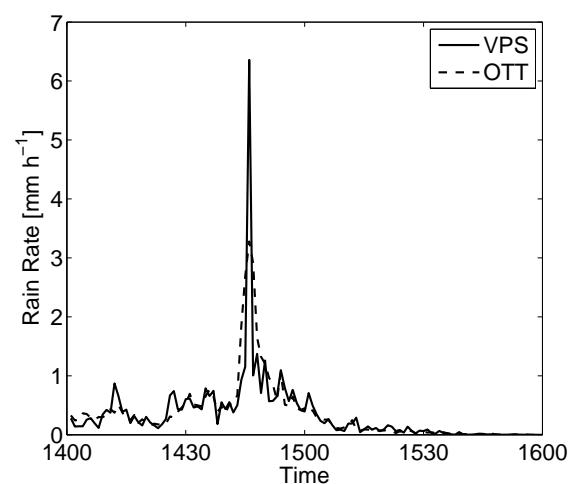

(a)

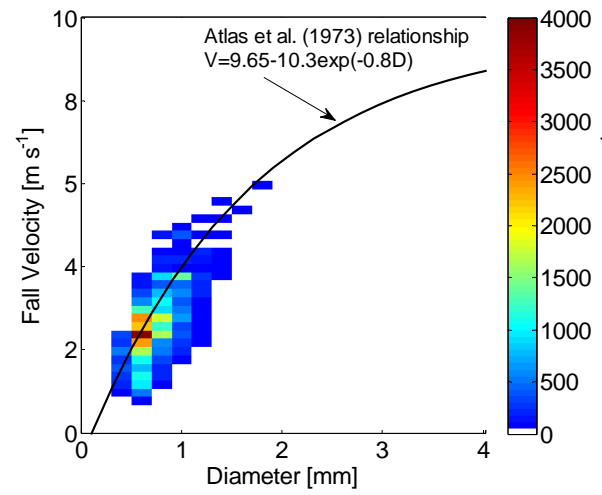

(c) fall velocity by VPS

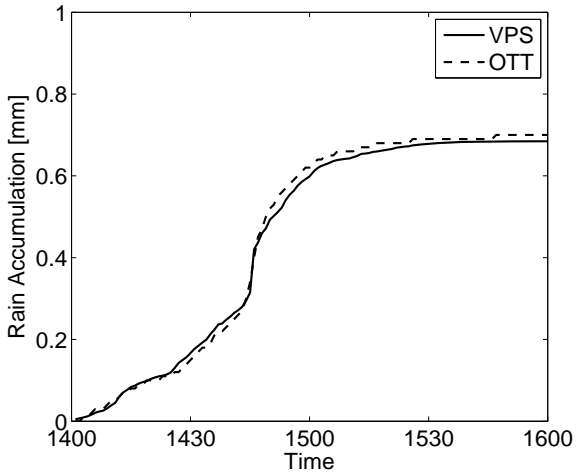

(b)

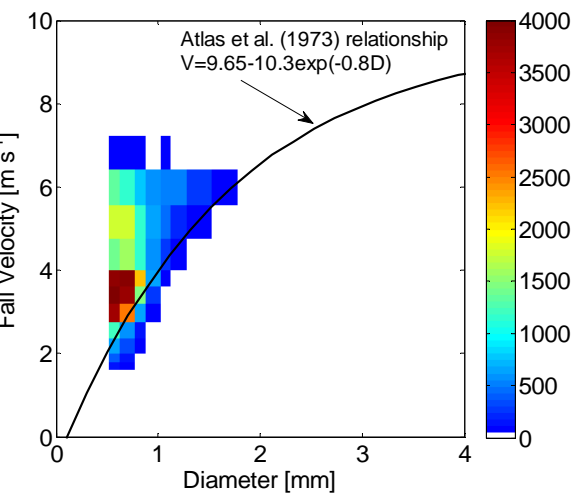

(d)fall velocity by OTT

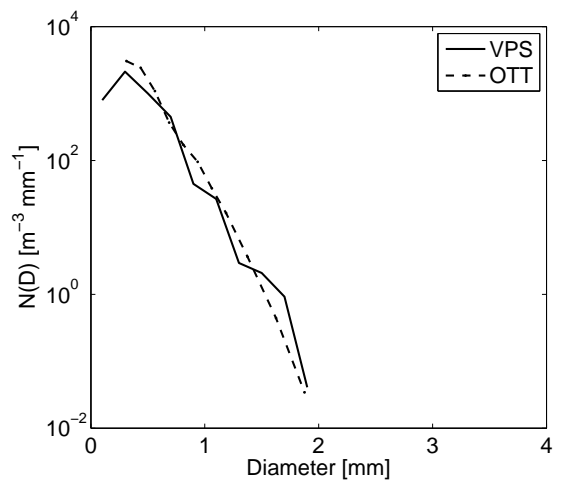

(e)

Figure 13. Rainfall observations from 14:00 to 17:00, 7 June 2013 (UTC).

time interval between two exposures and displacement of particles simultaneously; the drop size distribution, drop velocity distribution, precipitation intensity, and classification can be estimated by time integration. VPS can detect the hydrometeor from as small as $0.1 \mathrm{~mm}$ up to $20 \mathrm{~mm}$ with a resolution of $0.1 \mathrm{~mm}$ (size), $0.1 \mathrm{~m} \mathrm{~s}^{-1}$ (velocity), $0.1 \mathrm{~mm} \mathrm{~h}^{-1}$ (precipitation intensity).

From May 2013, several rainfall events were observed by VPS at Nanjing, China. Time series and statistical distributions of the particles collected during this period are discussed and compared with the results of an OTT PARSIVEL disdrometer in this paper. The observations have shown that the VPS can record the sharp images of raindrops in free fall, the data processing algorithms can calculate the size, axis ratio, fall velocity, and orientation of precipitation particles simultaneously, based on which the precipitation intensity, drop size distribution, velocity distribution, shape distribution, orientation distribution can be estimated. Thus besides the classical data products, a VPS system can produce more refined data products, which are important for 
interpreting weather radar data, investigating regional precipitation features, and studying the physical process of precipitation.

It should be noted that VPS basically represents projections of the drop shapes onto only one plane, and there are limitations on axis ratio measurements, particularly if drops are oscillating with significant components of the asymmetric modes. Given that the raindrops take on different characteristics during the falling process, some variables change with the time. Although high speed imaging techniques in the wind tunnel can examine the apparent characteristics of the drop images as time series to deduce the various oscillation modes, the oscillation of falling drops cannot be measured based on the images of VPS. There are several issues that require further research: (i) the performance of VPS in the natural environment, especially in the windy weather, should be examined thoroughly, and the inverse algorithm should be refined accordingly; (ii) more field experiments of VPS for snowfall and hail should be carried out; (iii) the comparisons of VPS with rain gauges, 2DVD, and PARSIVEL disdrometers can be made in the future.

Acknowledgements. This work is supported by the National Natural Science Foundation of China (grant no. 41327003, and 41205125). The VPS was developed with support from Ying EnTe environment technique Co.LTD, Nanjing, China.

Edited by: P. Stammes

\section{References}

Andsager, K., Beard, K. V., and Laird, N. F.: Laboratory measurements of axis ratios for large raindrops, J. Atmos. Sci., 56, 26732683, 1999.

Atlas, D., Srivastava, R. C., and Sekhon, R. S.: Doppler radar characteristics of precipitation at vertical incidence, Rev. Geophys. Space Phys., 11, 1-35, 1973.

Barthazy, E., Goke, S., Schefold, R., and Hogl, D.: An Optical Array Instrument for Shape and Fall Velocity Measurements of Hydrometeors, J. Atmos. Oceanic Technol., 21, 1400-1416, 2004.

Battaglia, A., Rustemeier, E., Tokay, A., Blahak, U., and Simmer, C.: PARSIVEL Snow Observations: A Critical Assessment, J. Atmos. Oceanic Technol., 27, 333-344, 2010.

Beard, K. V. and Chuang, C.: A New Model for the Equilibrium Shape of Raindrops, J. Atmos. Sci., 44, 1509-1524, 1987.

Chandrasekar, V., Cooper, W. A., and Bringi, V. N.: Axis Ratios and Oscillations of Raindrops, J. Atmos. Sci., 45, 1323-1333, 1988.

Huang, G. J., Bringi, V. N., and Thurai, M.: Orientation Angle Distributions of Drops after an 80-m Fall Using a 2D Video Disdrometer, J. Atmos. Oceanic Technol., 25, 1717-1723, 2008.

Jones, B. K., Saylor, J. R., and Bliven, L. F.: Single camera method to determine the optical axis of ellipsoid drops, Appl. Optics, 42, 972-978, 2003.
Joss, J. and Waldvogel, A.: Ein Spektrograph fur Niederschlagstrophen mit automatischer Auswertung, Pure Appl. Geophys, 68, 240-246, 1967.

Joss, J. and Waldvogel, A.: Comments on "Some Observations on the Joss-Waldvogel Rainfall Disdrometer”, J. Appl. Meteorol., 16, 112-113, 1977.

Kruger, A. and Krajewski, W. F.: Two-Dimensional Video Disdrometer: A Description, J. Atmos. Oceanic Technol., 19, 602-617, 2002.

Löffler-Mang, M. and Joss, J.: An Optical Disdrometer for Measuring Size and Velocity of Hydrometeors, J. Atmos. Oceanic Technol., 17, 130-139, 2000.

Liu, X. C., Gao, T. C., and Liu, L.: A comparison of rainfall measurements from multiple instruments, Atmos. Meas. Tech., 6, 1585-1595, doi:10.5194/amt-6-1585-2013, 2013.

Marzuki, Randeu, W. L., Kozu, T., Shimomai, T., Hashiguchi, H., and Schönhuber, M.: Raindrop axis ratios, fall velocities and size distribution over Sumatra from 2D-Video Disdrometer measurement, Atmos. Res., 119, 23-37, 2013.

Newman, A. J. and Kucera, P. A.: Presenting the Snowflake Video Imager (SVI), J. Atmos. Oceanic Technol., 26, 167-179, 2009.

Pruppacher, H. R. and Beard, K. V.: A wind tunnel investigation of the internal circulation and shape of water drops falling at termina lvelocity in air, Q. J. Roy. Meteorol. Soc., 96, 247-256, 1970.

Saylor, J. R. and Sivasubramanian, N. A.: Edge detection methods applied to the analysis of spherical raindrop images, Appl. Optics, 46, 5352-5367, 2007.

Saylor, J. R., Jones, B. K., and Bliven, L. F.: A method for increasing depth of field during droplet imaging, Rev. Scientific Instr., 73, 2422-2427, 2002.

Schönhuber, M., Lammer, G., and Randeu, W. L.: One decade of imaging precipitation measurement by $2 \mathrm{D}$-video-distrometer, Adv. Geosci., 10, 85-90, doi:10.5194/adgeo-10-85-2007, 2007.

Thurai, M. and Bringi, V. N.: Drop Axis Ratios from a 2D Video Disdrometer, J. Atmos. Oceanic Technol., 22, 966-978, 2005.

Thurai, M., Huang, G. J., Bringi, V. N., Randeu, W. L., and Schönhuber, M.: Drop shapes, model comparisons, and calculations of polarimetric radar parameters in rain, J. Atmos. Oceanic Technol., 24, 1019-1032, 2007.

Thurai, M., Bringi, V. N., and Petersen, W. A.: Rain microstructure retrievals using 2-D video disdrometer and C-band polarimetric radar, Adv. Geosci., 20, 13-18, doi:10.5194/adgeo-20-13-2009, 2009a.

Thurai, M., Szakall, M., Bringi, V. N., and Beard, K. V.: Drop Shapes and Axis Ratio Distributions: Comparison between 2D Video Disdrometer and Wind-Tunnel Measurements, J. Atmos. Oceanic Technol., 26, 1427-1432, 2009 b.

Thurai, M., Petersen, W. A., Tokay, A., Schultz, C., and Gatlin, P.: Drop size distribution comparisons between Parsivel and 2-D video disdrometers, Adv. Geosci., 30, 3-9, doi:10.5194/adgeo30-3-2011, 2011.

Tokay, A., Kruger, A., and Krajewski, W. F.: Comparison of drop size distribution measurements by impact and optical disdrometers, J. Appl. Meteorol., 40, 2083-2097, 2001. 